Enferm Bras 2020;19(3):261-67

https://doi.org/10.33233/eb.v19i3.4127

\title{
REVISÃo \\ Pré-eclâmpsia na gestação: ênfase na assistência de enfermagem
}

Rayani Silva Sarmento*, Wilton Medeiros da Silva*, Micaelly Araújo Gomes*, Liliane Noemia Torres de Melo**

*Graduados em Enfermagem, Faculdade Uninassau Caruaru, ${ }^{* *}$ Orientadora, Faculdade Uninassau Caruaru

Recebido em 14 de maio de 2020; aceito em 04 de junho de 2020.

Correspondência: Rayani Silva Sarmento, Rua Marquês de Olinda, 98, 55295-500 Garanhuns PE

Rayani Silva Sarmento: rayani_barros@hotmail.com

Wilton Medeiros da Silva: wiltonb@@hotmail.com

Micaelly Araújo Gomes: micaellysbu@gmail.com

Liliane Noemia Torres de Melo: liliane_melo@hotmail.com

\section{Resumo}

Introdução: Durante a gravidez podem ocorrer complicações, colocando em risco a vida do feto e da mãe, sendo uma dessas a pré-eclâmpsia, decorrente de níveis tensionais elevados relacionado à proteinúria, após a $20^{\underline{a}}$ semana. Objetivo: Analisar a assistência de Enfermagem em mulheres que apresentam quadros de pré-eclâmpsia. Métodos: Este estudo é de caráter descritivo, com uma abordagem qualitativa. Todo o referencial teórico é decorrente de uma revisão realizada no período entre 2000 e 2019, em três bancos de dados: Scielo, Medline e Google acadêmico. Resultados: $O$ trabalho do enfermeiro nos cuidados com a gestante são condutas que tem como ação o monitoramento do pré-natal e adoção de medidas preventivas e/ou terapêuticas que possam minimizar as complicações das gestantes. Conclusão: A atuação do enfermeiro é um importante instrumento na redução das complicações na pré-eclâmpsia, com ações voltadas à prevenção de agravos na gestação.

Palavras-chave: pré-eclâmpsia, assistência de enfermagem, fatores de risco, gestantes.

\section{Abstract \\ Pre-eclampsia during the pregnancy: emphasis on nursing care}

Introduction: Some complications may occur during the gestation, and it can put in risk the mother and unborn baby's lives. One of those complications being pre-eclampsia, which is a result of high blood pressure levels related to proteinuria, after the twentieth week. Aim: To analyze nursing care for women with pre-eclampsia. Methods: This study have descriptive feature, epidemiological with a qualitative approach. The entire theoretical reference is the result of a literature review published between 2000 and 2019 available in three databases: Scielo, Medlinee and Google Scholar. Results: The nurse's work in taking care of pregnant woman consists of actions including; the prenatal monitoring, adopting preventive measures and therapeutics that can minimize the complications of pregnant women. Conclusion: The nurse's performance is an important instrument to reduce the possibility of pre-eclampsia, with actions turned to prevention of complications during pregnancy.

Keywords: pre-eclampsia, nursing care, risk factors, pregnant women.

\section{Resumen}

\section{Preeclampsia en la gestación: énfasis en la asistencia de enfermería}

Introducción: Durante el embarazo pueden ocurrir complicaciones, poniendo en riesgo la vida del feto y de la madre, siendo una de esas la preeclampsia, causada por niveles tensionales elevados relacionados a la proteinuria, después de la $20^{\circ}$ semana. Objetivo: Analizar los cuidados de enfermería para mujeres con preeclampsia. Métodos: Este estudio es de carácter descriptivo, epidemiológico con un abordaje cualitativo. Todo el referencial teórico es el resultado de una revisión bibliográfica realizada en el período entre 2000 y 2019, en tres bancos de datos: 
Scielo, Medline y Google académico. Resultados: El trabajo del enfermero en los cuidados con la embarazada son conductas que tienen como acción el monitoreo del prenatal y adopción de medidas preventivas y/o terapéuticas que pueden minimizar las complicaciones de la embarazada. Conclusión: La actuación del enfermero es un importante instrumento en la reducción de las complicaciones en la preeclampsia, con acciones enfocadas a la prevención de problemas en la gestación.

Palabras-clave: preeclampsia, asistencia en enfermería, factores de riesgo en embarazadas.

Introdução

Entre os anos de 2000 e 2019 a saúde da mulher e da criança vem sendo mundialmente estudada e, no Brasil, é considerada uma prioridade dos grupos de estudos [1]. Porém, mesmo com esse cuidado e estudo, o número de mortes provenientes de complicações da gestação e do parto ainda é ainda elevado. Entre as diferentes complicações, destacam-se aqueles decorrentes da gestação de alto risco [2,3]. A gestação de alto risco ocorre quando a gestante apresenta alguma doença ou condição sociobiológica como a hipertensão arterial, diabetes, alcoolismo, obesidade entre outras, que complica a evolução da gravidez, em que muitas vezes esse risco pode levar à morte materna [4].

A pré-eclâmpsia (PE) é caracterizada pela presença de níveis tensionais elevados, como consequência de uma hipertensão durante a gestação, após a $20^{\text {a }}$ semana relacionada à proteinúria, sendo responsável por grande parte da interrupção prematura da gestação $[5,6]$.

As mulheres com PE não se sentem doentes até a condição de se tornar muito grave, oferecendo risco de vida. Por isso, é necessário que seja realizada a detecção precoce pelos profissionais de saúde e, consequentemente, tratamento de forma eficaz [7], para evitar a evolução de uma forma grave da doença que coloca em risco a vida da mãe e do feto [8].

O Ministério da Saúde destaca a importância de uma abordagem integral às mulheres e preconiza o manejo adequado de situações de vulnerabilidade relacionadas ao processo saúdedoença, sejam elas individuais, sociais e/ou programáticas. A interdependência entre vulnerabilidade programática e a gestação de alto risco envolve o acesso aos serviços de saúde e a oportunidade de informações advindas de profissionais da área [9].

Dentre os profissionais de saúde capacitados para prestar assistência adequada, destaca-se o enfermeiro, sendo um dos principais objetivos de trabalho o cuidar. Esse profissional desempenha um papel fundamental no cuidado e na educação de mulheres em risco. É indispensável uma enfermagem de qualidade a fim de prestar assistência às mulheres e suas famílias, visando a detecção precoce de fatores de risco e o melhor gerenciamento clínico de doenças [10].

Considera-se como assistência em enfermagem fazer pelo ser humano aquilo que ele não pode por si mesmo; como também ajudar ou auxiliar quando parcialmente impossibilitado de se autocuidar; orientar ou ensinar; supervisionar ou encaminhar a outros profissionais. Nesse sentido, deve-se destacar que o tratamento de pacientes com pré-eclâmpsia depende muito da atuação dos enfermeiros [11].

Mesmo que interligada e complementada por outros saberes profissionais, a enfermagem pode ser amplamente definida como a ciência do cuidado integral e integrador em saúde, tanto no sentido de assistir e coordenar as práticas de cuidado, quanto no sentido de promover e proteger a saúde dos indivíduos, famílias e comunidades [12].

Este artigo traz para o centro das discussões a conduta dos enfermeiros frente a gestantes portadoras de pré-eclâmpsia. A escolha do tema justifica-se pelo fato de ainda existirem muitos profissionais de enfermagem com atitudes generalistas, que não buscam capacitar-se para assistência humanizada. Além disso, estudos na área são escassos, embora seja tão importante para sociedade.

Sendo assim, este estudo tem como objetivo estudar o papel do enfermeiro na prevenção de gestantes que apresentam quadros de pré-eclâmpsia. Para isso, investiga a conduta do enfermeiro frente a doença, evidenciando as práticas mais eficazes no tratamento das mulheres que apresentam o quadro.

Material e métodos

O estudo teve caráter descritivo com abordagem qualitativa. Todo o referencial teórico foi decorrente de uma revisão bibliográfica realizada em três bancos de dados: Scielo, Medline e Google acadêmico. Para a busca utilizou-se os seguintes descritores: "Pré-eclâmpsia"; 
"Assistência de Enfermagem", "Fatores de risco e gestantes." Foram selecionados 38 trabalhos, sendo analisados 27 destes para compor o estudo. Adotou-se como critérios de inclusão, artigos que abordam o tema, com disponibilidade de texto completo. Monografias, teses e artigos publicados antes de 2000 foram excluídos da análise.

Resultados e discussão

Atenção integral a saúde da mulher

A situação de saúde envolve diversos aspectos da vida, como a relação com o meio ambiente, o lazer, a alimentação e as condições de trabalho, moradia e renda. No caso das mulheres, os problemas são agravados pela discriminação nas relações de trabalho e a sobrecarga com as responsabilidades com o trabalho doméstico. Outras variáveis como raça, etnia e situação de pobreza realçam ainda mais as desigualdades [14].

De acordo com o Ministério da Saúde, a população feminina comporta a maioria da população brasileira $(50,77 \%)$ e são principais usuárias do Sistema Único de Saúde (SUS). As mulheres frequentam os serviços de saúde para o seu próprio atendimento, mas, sobretudo, acompanhando crianças e outros familiares, pessoas idosas, vizinhos, amigos, entre outros [14].

Encontram-se na literatura vários conceitos sobre saúde da mulher, em que há concepções mais restritas que abordam apenas aspectos da biologia e anatomia do corpo feminino e outras mais amplas que interagem com dimensões dos direitos humanos e questões relacionadas à cidadania. Nas concepções mais restritas, o corpo da mulher é visto apenas na sua função reprodutiva e a maternidade torna-se sua principal característica. A saúde da mulher limita-se à saúde materna ou à ausência de enfermidade associada ao processo de reprodução biológica. Nesse caso, estão excluídos os direitos sexuais e as questões de gênero, em que se refere ao conjunto de relações, papéis, religiões e atitudes que determinam a diferença entre 0 sexo, em que muitas vezes na sociedade essas são desiguais [15].

Como consequência, a humanização e qualificação a atenção em saúde é primordial, aprendendo a compartilhar saberes e reconhecê-los. A atenção humanizada e de boa qualidade implica no estabelecimento de relações entre os sujeitos, seres semelhantes, ainda que possam apresentar-se muito distintos conforme suas condições sociais, raciais, étnicas, culturais e de gênero [14].

\section{Pré-eclâmpsia}

A pré-eclâmpsia é definida pela presença de níveis tensionais elevados na gravidez, após a $20^{\underline{a}}$ semana, relacionados à proteinúria, sendo responsável por grande parte das indicações de interrupção prematura da gestação [16]. De acordo com os últimos dados divulgados pelo Departamento de Informática do Sistema Único de Saúde (DATASUS), as síndromes hipertensivas causaram 325 óbitos, representando $20 \%$ das causas de óbitos maternos, e $56 \%$ destes aconteceram no período de gravidez [17].

A obesidade em gestantes é um importante fator de risco para o desenvolvimento da PE, principalmente na forma grave, trazendo complicações para o feto [18]. Além disso, a probabilidade de ocorrer uma PE aumenta com a idade materna, técnicas de reprodução assistida, assim como comorbidades que predispõe a PE como diabetes, hipertensão e doenças renais [19].

"A pré-eclâmpsia compromete todos os órgãos e sistemas maternos e, com maior intensidade, os sistemas vascular, hepático, renal e cerebral. Sendo definida como grave pela presença de um ou mais dos seguintes critérios: Pressão arterial $\geq 160 / 110 \mathrm{mmHg}$; Proteinúria $\geq 2 \mathrm{~g} / 24$ horas; Creatinina sérica > 1,2 mg\%; Oligúria < $500 \mathrm{ml} / 24$ horas; Distúrbios visuais e/ou cerebrais; Edema pulmonar ou cianose; Dor epigástrica ou no quadrante superior direito do abdome; Disfunção hepática; Plaquetopenia; Eclâmpsia; Restrição de crescimento fetal [20: 269]"

As crianças que nascem de gestações acometidas de pré-eclâmpsia apresentam maior risco de síndromes metabólicas, doenças cardiovasculares e hipertensão sistêmica precoce [20]. 
A evolução do quadro de uma paciente com pré-eclâmpsia pode ocorrer em ritmos muito diferentes. Umas se estabilizam até o fim da gestação, outras tem a situação deteriorada progressivamente ao longo de semanas, e algumas apresentam sinais de gravidade em dias ou até mesmo em horas [21].

\section{O papel do enfermeiro na prevenção da pré-eclâmpsia}

A atuação do enfermeiro nos cuidados com a gestante são condutas que tem como ação o monitoramento do pré-natal e adoção de medidas preventivas e/ou terapêuticas que possam minimizar complicações. Mostra-se, assim, como elemento ativo, ao executar a assistência à gestante no pré-natal e até mesmo da gravidez, durante as consultas de planejamento familiar na busca de identificar fatores de risco e doenças que ofereçam alguma complicação durante a gestação, principalmente nas populações de maior vulnerabilidade [21].

Os cuidados de enfermagem específicos a mulheres com pré-eclâmpsia e/ou eclampsia são capazes de reduzir complicações e taxas de morbimortalidade. A assistência de enfermagem descrita na presente revisão abrange, principalmente, exame físico criterioso; identificação precoce de sinais de pré-eclâmpsia/eclampsia; acompanhamento de exames laboratoriais; avaliação fetal; treinamentos dos profissionais, incluindo necessidade de educação continuada; padronização do atendimento a partir de instrumentos; aferição da PA com manguito adequado à circunferência do braço; velocidade lenta de desinsuflação da coluna de mercúrio $(\leq 2 \mathrm{mmHg})$; necessidade da padronização da técnica de aferição da PA; identificação e tratamento precoce da crise hipertensiva mediante protocolos institucionais; bem como a revisão de casos e processos de trabalho [21].

Criar e adotar protocolos de cuidado pautados em evidência científica na prática clínica do enfermeiro, diariamente, pode ser útil para nortear o processo de tomada de decisão e garantir a prestação de uma assistência de qualidade e segura [22].

Conhecer a atuação da Enfermagem implementada ao tratamento da Pré-Eclâmpsia é importante para garantir a qualidade do atendimento ofertado. Muitas gestantes só descobrem a patologia no momento de internação, o que gera inúmeros desconfortos emocionais para elas que já passam por alterações hormonais que afetam ainda mais seus sentimentos. Esse desconhecimento sobre a doença poderia ser evitado por meio de assistência pré-natal efetiva, com acompanhamento contínuo, tendo em vista que a assistência durante a gestação pautada em orientações e correção de dúvidas faz com que a gestante se envolva no processo de autocuidado, proporcionando uma gestação saudável [23].

A consulta de enfermagem, na atenção primária à saúde, é realizada de acordo com o roteiro estabelecido pelo Ministério da Saúde. Esse roteiro proporciona a orientação adequada e que favorece a abordagem apropriada das necessidades peculiares das mulheres com quem os profissionais interagem em consultas no pré-natal, nas Unidades Básicas de Saúde que devem ser a porta de entrada de referência da gestante [21].

A gravidez de alto risco precisa de uma assistência de enfermagem pautada em métodos científicos que fazem o cuidado padrão ouro para a saúde da paciente [23]. O uso de uma abordagem certa e humanizada, em que a paciente é o foco principal do cuidado, levará uma melhora dos resultados, pois irá proporcionar uma maior capacidade no atendimento, diminuindo gastos devido à redução de morbimortalidade; além de promover um padrão de confiança e segurança às instituições de saúde [21,24].

Enfatiza-se, ainda, que em razão dos enfermeiros estarem mais presentes no cuidado ofertado às mães e aos recém-nascidos, são capazes de fornecer a vigilância contínua necessária a essa doença complicada e desafiadora para a saúde pública [10].

\section{Práticas mais eficazes dos enfermeiros diante da pré-eclâmpsia}

As atribuições do enfermeiro consistem em assistir o usuário no planejamento da gravidez, no diagnóstico, nas rotinas da atenção ao pré-natal com o plano da primeira consulta, no plano das consultas de retorno, frequência das consultas, imunização, ações educativas, visitas domiciliares, encaminhamentos e transferências, sempre com o propósito de oferecer subsídios centrados no cuidado e a comunicação, sendo um recurso indispensável para a assistência à saúde, com vistas ao estabelecimento de confiança e a vinculação do usuário ao profissional [21]. 
Independentemente do nível de complexidade de atendimento, o profissional enfermeiro tem o dever de participar do processo de avaliação do "fazer da Enfermagem" de forma a contribuir com a assistência prestada nos serviços de saúde [25].

Embora o tratamento de enfermagem se caracterize pela atuação do enfermeiro, orientando, supervisionando, ajudando ou encaminhando a paciente, percebe-se também que a prevenção existe através de ações educativas específicas. Alguns fatores são importantes na decisão terapêutica, como o tipo de síndrome hipertensiva, a gravidade da doença, o período gestacional de surgimento do quadro e a manutenção da homeostase mãe-feto. A tabela a seguir mostra alguns tratamentos para as mulheres que apresentam o quadro [26].

Tabela I - Resumo do tratamento da pré-eclâmpsia.

\begin{tabular}{|c|c|}
\hline Tratamento & Recomendações \\
\hline Resolução da gravidez & $\begin{array}{l}\text { Recomendada de acordo com a idade gestacional, gravidade da } \\
\text { pré-eclâmpsia, bem-estar fetal e presença ou não de } \\
\text { complicações. }\end{array}$ \\
\hline Conduta conservadora & $\begin{array}{l}\text { Recomendada para melhora das condições perinatais em } \\
\text { gestações antes do termo. Razoável segurança materna para um } \\
\text { grupo sele cionado de pacientes com pré-eclâmpsia grave e } \\
\text { condições materno-fetais estáveis. }\end{array}$ \\
\hline Corticoterapia & $\begin{array}{l}\text { Recomendada em pacientes com idade gestacional abaixo de } 34 \\
\text { seman as completas. Ausência de riscos maternos adicionais para } \\
\text { morte, corioamnionite ou sepse puerperal, como também } \\
\text { diminuição de riscos provenientes da pre maturidade. }\end{array}$ \\
\hline Repouso & $\begin{array}{l}\text { Há poucas evidências demonstrando alguma dife rença entre } \\
\text { repouso e atividade normal. O esforço físico deverá ser } \\
\text { desencorajado em pacientes com pré-eclâmpsia. }\end{array}$ \\
\hline Expansores plasmáticos & $\begin{array}{l}\text { Não há evidência suficiente para que sejam recomendados de } \\
\text { rotina. }\end{array}$ \\
\hline Sulfato de magnésio & $\begin{array}{l}\text { Recomendado em gestantes com potencial risco de eclâmpsia. } \\
\text { Deve ser administrado a todas as pacientes com pré-eclâmpsia } \\
\text { grave (NNT=63) e ter o uso discutido de acordo com o quadro } \\
\text { clínico em gestantes com pré-e clâmpsia leve (NNT=110). }\end{array}$ \\
\hline Tratamento Anti-hipertensivo & $\begin{array}{l}\text { Recomendado para tratamento agudo dos picos hipertensivos em } \\
\text { pacientes com pré-eclâmpsia. O tratamento de manutenção ainda } \\
\text { é alvo de controvérsias. }\end{array}$ \\
\hline Droga anti-hipertensiva & $\begin{array}{l}\text { Na emergência, o clínico deve usar a droga com a qual tenha } \\
\text { maior familiaridade e experiência. As opções são nifedipina (oral), } \\
\text { hidralazina (injetáv el) e labetalol (injetáv el). Para tratamento de } \\
\text { manutenção, estão disponíveis por via oral alfametild opa, } \\
\text { labetalol, nifedipina, pindolol e atenolol. }\end{array}$ \\
\hline Antecipação do parto & $\begin{array}{l}\text { O único tratamento efetivo da pré-eclâmpsia/eclâmpsia, } \\
\text { determinando a "cura" do proce sso patológico, é o parto. Todas } \\
\text { as outras modalidades terapêuticas destinam-se a manter estável } \\
\text { o quadro clínico materno e a vigiar a vitalidade fetal enquanto se } \\
\text { aguarda a maturação pulmonar do concepto (permitindo melhores } \\
\text { taxas de sobrevida neonatal) e, algumas vezes, o pre paro } \\
\text { cervical }\end{array}$ \\
\hline Via de parto & $\begin{array}{l}\text { A interrupção da gravidez em pacientes com pré-eclâmpsia pode } \\
\text { ser programada por cesárea eletiva ou indução do trabalho de } \\
\text { parto. }\end{array}$ \\
\hline
\end{tabular}

O propósito do tratamento é prevenir as complicações materno-fetais, como, por exemplo, descolamento prematuro de placenta, acidente vascular cerebral, insuficiência renal, edema agudo de pulmão, aumento do quadro clínico para pré-eclâmpsia grave, síndrome Hellp e eclampsia. Em relação ao feto, previne o parto prematuro e o desconforto respiratório do recémnascido [27].

\section{Conclusão}

O estudo descreveu a assistência dos enfermeiros, mostrando que são capazes de reduzir complicações e taxas de mortalidade materno/infantil. As ações humanísticas realizadas pelos enfermeiros buscam uma assistência com excelência, pois agem de forma acolhedora 
preservando a vida da paciente, que além de prestar uma assistência competente à saúde materna, inclui a avaliação da vitalidade fetal, por meio de exames entre outros procedimentos.

Assim, a atuação do enfermeiro é um importante instrumento na redução das complicações decorrentes da pré-eclâmpsia, proporcionando melhoria na assistência de enfermagem através de mudanças na prática clínica, com ações voltadas à prevenção de agravos na gestação, para com isso pode proporcionar uma diminuição dos índices de morbimortalidade.

Destaca-se a necessidade de estudos sobre a temática com rigor metodológico, buscando fornecer ao enfermeiro subsídios para assistência de enfermagem, focando mais nesse assunto; incentivando estudantes e atuantes da área da saúde desenvolverem pesquisas e trabalhos acadêmicos que abranjam o papel do enfermeiro a portadoras de pré-eclâmpsia. É importante ofertar Educação Permanente com capacitação dos profissionais de saúde nesse tema para, assim, melhorar cada vez mais os atendimentos, e despertar o pensamento crítico do enfermeiro quanto a sua conduta a pacientes com pré-eclâmpsia.

1. Ministério da Saúde. Área Técnica de Saúde da Mulher. Plano de ação para o período 2004 a 2007. [citado 2019 Dez 12]. Disponível em:

http://bvsms.saude.gov.br/bvs/publicacoes/politica nac atencao mulher.pdf

2. Costa LD, Cura CC, Perondi AR, França VF, Bortoloti DS. Perfil epidemiológico de gestantes de alto risco, Cogitare Enferm 2016;21(2):1-8.

3. Peixoto CR, Freitas LV, Teles LMR, Campos FC, De Paula PF, Damasceno AKC. O pré-natal na atenção primária: o ponto de partida para reorganização da assistência obstétrica. Rev Enferm UERJ 2011;19(2):286-91.

4. Luz BG, Soares LT, Grillo VTRS, Viola MB, Laporte IC, Bino DBM. O perfil das gestantes de alto risco acompanhadas no pré-natal da policlínica de Divinópolis-MG, no biênio 2013-14. J Health Biol Sci 2015;3(3):137-43.

5. Melo BCP. Perfil epidemiológico e evolução clínica pós-parto na pré-eclâmpsia grave. AMB Rev Assoc Med Bras 2009;55(2):123-43.

6. Freire CMV, Tedoldi CL. Hipertensão arterial na gestação. Arq Bras Cardiol 2009;93(6):110-78.

7. Magalhães DL. Assistência da Enfermagem na atenção a Pré- Eclâmpsia/Eclâmpsia. Centro Universitário de Brasilia 2015;5(3):7-11.

8. Spindola T, Lima GLS, Cavalcanti RL. A ocorrência de pré-eclâmpsia em primigestas mulheres acompanhadas no pré-natal de um hospital universitário. Fundam Care online 2015;5(3):235-44.

9. Ministério da Saúde. Secretaria de Políticas de Saúde. Manual gestação de alto risco 2010 [acesso em 12 dez 2019]. Disponível em:

http://bvsms.saude.gov.br/bvs/publicacoes/manualgestacaodealtorisco.pdf

10. Ferreira ETM, Moura NS, Gomes MLS, Silva EG, Guerreiro MGS, Oriá MOB. Características maternas e fatores de risco para pré-eclâmpsia em gestantes. Rev Rene 2019;20:403-27. https://doi.org/.10.15253/2175-6783.20192040327

11. Cunha KJB, Oliveira JO, Nery IS. Assistência de Enfermagem na opinião das mulheres com pré-eclâmpsia. Esc Anna Nery Rev de Enferm 2009;11(2):554-60.

12. Backes DS, Backes MS, Erdmann AL, Andreas Büscher. O papel profissional do enfermeiro no Sistema Único de Saúde: da saúde comunitária à estratégia de saúde da família. Ciênc Saúde Coletiva 2012;17(1):141-23. https://doi.org/10.1590/S141381232012000100024

13. Lima-Costa MFF, Guerra HL, Barreto SM, Guimarães RM. Diagnóstico de saúde da população idosa brasileira: um estudo da mortalidade e das internações hospitalares públicas. Informe Epidemiológico do SUS 2003. https://doi.org/10.5123/S010416732000000100003

14. Coelho MRS. Atenção básica à saúde da mulher: subsídios para a elaboração do manual do gestor municipal [Dissertação]. Instituto de Saúde Coletiva, Universidade Federal da Bahia, Salvador, 2003. [citado 2019 Dez 12]. Disponível em: https://bvsms.saude.gov.br/bvs/publicacoes/politica nac atencao mulher.pdf

15. Freire CMV, Tedoldi CL. Hipertensão arterial na gestação. Arq Bras Cardiol 2009;93(6):66-82. https://doi.org/10.1590/S0066-782X2009001300017 
16. Ministério da Saúde. Departamento de Informática do Sistema Único de Saúde (DATASUS). Óbitos de mulheres em idade fértil e óbitos maternos 2011 [citado 2019 Dez 13]. Disponível em:

https://www.scielo.br/scielo.php?script=sci arttext\&pid=S003471672018000700677\&ln $\mathrm{q}=$ en\&nrm=iso/\&tlng=pt

17. Townsend $R, O$ 'brien $P$, Khalil A. Current best practice in the management of hypertensive disorders in pregnancy. Integrated Blood Pressure Control 2016;9:79-94. https://doi.org/10.2147/ibpc.s77344

18. Marques DSM, Siqueira HGR, Cruz MA, Vieira MSV, Apolônio RSPT. A relação entre pré-eclâmpsia e obesidade: Uma revisão integrativa. Rev Caderno de Medicina 2019;2(2):56-62.

19. Peraçoli JR, José C, Parpinelli MA. Síndromes hipertensivas da gestação: identificação de casos graves. Rev Bras Ginecol Obstet 2005;27(10):627-34. https://doi.org/10.1590/S0100-72032005001000010

20. Ferreira MBG, Silveira CF, Silva SR, Souza DJ, Ruiz MT. Nursing care for women with pre-eclampsia and/or eclampsia: integrative review. Rev Esc Enferm USP 2016;50(2):320-30. https://doi.org/10.1590/S0080-623420160000200020

21. Brasil Ministério da Saúde. Departamento de Atenção Básica. Estratégias para o cuidado da pessoa com doença crônica: hipertensão arterial sistêmica. Brasília 2013. [citado 2019 Dez 13]. Disponível em: http://bvsms.saude.gov.br/bvs/publicacoes/manual pre natal puerperio 3ed.pdf

22. Ferreira MBG, Silveira CF, Silva SR, Souza DJ, Ruiz MT. Nursing care for women with pre-eclampsia and/or eclampsia: integrative review. Rev Esc Enferm USP 2016;50(2):320-30. https://doi.org/10.1590/S0080-623420160000200020

23. Santana RS, Costa ACRR, Fontes FLL, Carvalho FR, Moura FF, Duarte JM, et al. Importância do conhecimento sobre sinais e sintomas da pré-eclâmpsia para implementação dos cuidados de Enfermagem: Revista Eletrônica Acervo Saúde 2019;11(15):1-6. https://doi.org/10.25248/reas.e1425.2019

24. Barbosa TLA, Gomes LMX, Dias OV. O pré-natal realizado pelo enfermeiro: a satisfação das gestantes. Cogitare Enferm 2011;16(1):29-35. https://doi.org/10.5380/ce.v16i1.21108

25. Edward KL, Mills C. A hospital nursing research enhancement model. J Contin Educ Nurs 2013;44(10):447-54. https://doi.org/ 10.3928/00220124-20130801-45

26. Noronha NC, Souza ASR, Amorim MMR. Tratamento da pré-eclâmpsia baseado em evidências. Rev Bras Ginecol Obstet 2010;32(9):459-68. https://doi.org/10.1590/S010072032010000900008

27. Kahhale S, Francisco RPV, Zugaib M. Pré-eclampsia. Rev Med (São Paulo) 2018;97(2):226-34. https://doi.org/10.11606/issn.16799836.v97i2p226-234 